\title{
Are sesquiterpene lactones the elusive KARRIKIN-INSENSITIVE2 ligand?
}

\author{
Mehran Rahimi $^{1} \cdot$ Harro Bouwmeester $^{1}$ B
}

Received: 25 September 2020 / Accepted: 8 January 2021 / Published online: 1 February 2021

(c) The Author(s) 2021

\begin{abstract}
Main conclusion The sunflower sesquiterpene lactones 8-epixanthatin and tomentosin can bind to the hydrophobic pocket of sunflower KAI2 with an affinity much higher than for the exogenous ligand KAR.
\end{abstract}

\begin{abstract}
Sesquiterpene lactones (STLs) are secondary plant metabolites with a wide range of biological, such as antimicrobial, activities. Intriguingly, the STLs have also been implicated in plant development: in several Asteraceae, STL levels correlate with the photo-inhibition of hypocotyl elongation. Although this effect was suggested to be due to auxin transport inhibition, there is no structural-functional evidence for this claim. Intriguingly, the light-induced inhibition of hypocotyl elongation in Arabidopsis has been ascribed to HYPOSENSITIVE TO LIGHT/KARRIKIN-INSENSITIVE2 (HTL/KAI2) signaling. KAI2 was discovered because of its affinity to the smoke-derived karrikin (KAR), though it is generally assumed that KAI2 has another, endogenous but so far elusive, ligand rather than the exogenous KARs. Here, we postulate that the effect of STLs on hypocotyl elongation is mediated through KAI2 signaling. To support this hypothesis, we have generated homology models of the sunflower KAI2s (HaKAI2s) and used them for molecular docking studies with STLs. Our results show that particularly two sunflower STLs, 8-epixanthatin and tomentosin, can bind to the hydrophobic pockets of HaKAI2s with high affinity. Our results are in line with a recent study, showing that these two STLs accumulate in the light-exposed hypocotyls of sunflower. This finding sheds light on the effect of STLs in hypocotyl elongation that has been reported for many decades but without conclusive insight in the elusive mechanism underlying this effect.
\end{abstract}

Keywords 3D structure models $\cdot 8$-Epixanthatin $\cdot$ Germination $\cdot$ Hypocotyl elongation $\cdot$ KAI2 signaling $\cdot$ Strigolactone

Sesquiterpene lactones (STLs) are secondary plant metabolites with a wide range of biological, such as anti-microbial, activities (Spring and Hager 1982; Chadwick et al. 2013; Liu et al. 2018). Intriguingly, the STLs have also been implicated in plant development: particularly in sunflower, STL levels correlate with the photo-inhibition of hypocotyl elongation (Spring and Hager 1982; Yokotani-Tomita et al. 1999; Arai

Communicated by Dorothea Bartels.

Harro Bouwmeester

h.j.bouwmeester@uva.nl

Mehran Rahimi

m.rahimi@uva.nl

1 Plant Hormone Biology Group, Green Life Sciences Cluster, Swammerdam Institute for Life Science, University of Amsterdam, Science Park 904, 1098 XH Amsterdam, The Netherlands et al. 2013). Although this effect was suggested to be due to auxin transport inhibition, there is no structural-functional evidence for this claim (Arai et al. 2013; Ueda et al. 2013). Intriguingly, the light-induced inhibition of hypocotyl elongation in Arabidopsis has been ascribed to HYPOSENSITIVE TO LIGHT/KARRIKIN-INSENSITIVE2 (HTL/ KAI2) signaling (Sun and Ni 2011; Waters and Smith 2013). KAI2 was discovered because of its affinity to the smokederived karrikin (KAR), though it is generally assumed that KAI2 has another, endogenous but so far elusive, ligand rather than the exogenous KARs (Conn and Nelson 2016). Here, we postulate that the effect of STLs on hypocotyl elongation is mediated through KAI2 signaling. To support this hypothesis, we have generated homology models of the sunflower KAI2s (HaKAI2s) and used them for molecular docking studies with STLs. Our results show that particularly two sunflower STLs, 8-epixanthatin and tomentosin, can bind to the hydrophobic pockets of HaKAI2s with high 
affinity. Our results are in line with a recent study, showing that these two STLs accumulate in the light-exposed hypocotyls of sunflower (Spring et al. 2020). This finding sheds light on the effect of STLs in hypocotyl elongation that has been reported for many decades but without conclusive insight in the elusive mechanism underlying this effect.

A number of studies showed that in sunflower, exposure to light results in an increase in the concentration of STLs in the hypocotyl, which correlates with the inhibition of elongation (Spring and Hager 1982; Yokotani-Tomita et al. 1999; Arai et al. 2013; Ueda et al. 2013). Moreover, accumulation of the STL, 8-epixanthatin, in the blue light-exposed side of sunflower hypocotyls was demonstrated and suggested to inhibit elongation, thus causing curvature toward the light. And finally, the application of exogenous STLs such as 8-epixanthatin significantly reduces the elongation of hypocotyls (Yokotani-Tomita et al. 1999).

The inhibition of hypocotyl elongation under light is also a well-known characteristic of KAR perception by plants (Sun and Ni 2011; Waters and Smith 2013). KARs are compounds derived from smoke that inhibit the hypocotyl elongation of Arabidopsis thaliana under blue and red light. KARs bind to KAI2, and hence induce seed germination and inhibit hypocotyl elongation (Nelson et al. 2010; Waters and Smith 2013). There is thus a striking similarity between the hypocotyl elongation inhibitory effects reported for STLs and KARs. Studies on the Arabidopsis kai2-2 mutant-that has an increased hypocotyl length-have suggested that KAI2 has an unknown endogenous ligand, coined KAI2 ligand (KL) (Conn and Nelson 2016). Most likely, KL acts in a similar fashion as KARs to activate KAI2 signaling, resulting in the same phenotypes. It is important to note that all efforts to identify KL have so far failed.

Over the past few years, a number of studies on the root parasitic Orobanchaceae showed that they have multiple copies of KAI2/HTLs. In Striga hermonthica, for example, the most studied parasitic plant species in this family, KAI2 underwent extensive gene duplication and it has at least 11 HTLs. Intriguingly, these receptors were shown to perceive exogenous signals, the so-called germination stimulants that are exuded from the roots of their host plant, which ensures that germination occurs near a host root (Conn et al. 2015; Toh et al. 2015; Bouwmeester et al. 2020). The germination stimulants for most parasitic plant species belong to the strigolactones, an intriguing class of compounds with endogenous, hormonal, roles as well as rhizosphere signaling activity (Bouwmeester et al. 2020). However, the germination of Orobanche cumana, a root parasitic plant that highly specifically parasitizes sunflower, was shown to be primarily induced by STLs (Joel et al. 2011; Raupp and Spring 2013). The root exudate of sunflower contains STLs such as dehydrocostuslactone, costunolide, tomentosin, and 8-epixanthatin (Joel et al. 2011; Raupp and Spring 2013).
This strongly suggest that one or more STLs can bind to one of the $O$. cumana KAI2/HTL receptors and induce KAI2/ HTL signaling and thus germination.

A recent study showed that the exposure of sunflower cotyledons to blue light results in a fast accumulation $(<2 \mathrm{~h})$ of 8-epixanthatin and tomentosin in the hypocotyl, which continues until reaching a maximum at $72 \mathrm{~h}$ (Spring et al. 2020). The other STLs, dehydrocostus lactone (which is abundantly present in roots) and costunolide, were only present in trace amounts and their concentrations did not change over time. This suggests that particularly 8 -epixanthatin and tomentosin are responsible for the inhibition of hypocotyl elongation. The four STLs are also present in dry sunflower seeds, although in very low concentration. Considering the role of KAI2 signaling in germination, they may therefore also be involved in (sunflower) seed germination.

Based on all the above, we postulate that the STLs, 8 -epixanthatin and tomentosin, in sunflower are the main ligands of HaKAI2s. To test our hypothesis, we identified two KAI2 homologs from the annotated genome sequence of $H$. annuus, and performed homology modeling using YASARA version 19.12.14 (Fig. 1a, b). The percentage identity of HaKAI2a and HAKAI2b is $86 \%$ (233 out of 279 amino acid residues are identical). The 3D structures of the four STLs were obtained from the PubChem Compound library as sdf files. For molecular docking, we used the Autodock Vina algorithm integrated in YASARA using the protein models with the highest $Z$ Score.

Molecular docking showed that 8-epixanthatin and tomentosin fit into the hydrophobic-binding pockets of HalKAI2a and b (Fig. 1c, f) with high binding affinity (Kd of 3.2 and $2.7 \mu \mathrm{M}$ for 8-epixanthatin and 6.0 and $6.2 \mu \mathrm{M}$ for tomentosin for HaKAI2a and b, respectively). The hydrophobic amino acid residues involved in the binding of the STLs in the pocket are depicted in Fig. 1c, d. Dehydrocostus lactone and costunolide do not fit properly in the hydrophobic pockets of the modeled receptors. As expected, also KARs (KAR1 and 2) bind the hydrophobic pockets of HalKAI2a, but with $c$. 25- to 50-fold lower affinity than the STLs (Kd of $165 \mu \mathrm{M}$ for KAR1 and $195 \mu \mathrm{M}$ for KAR2). Surprisingly, Arabidopsis KAI2 also strongly binds 8-epixanthatin and tomentosin with estimated Kd values of about 1 and $2 \mu \mathrm{M}$, respectively, compared with a $\mathrm{Kd}$ value for KAR1 of $57 \mu \mathrm{M}$, a significantly lower affinity than for the STLs. The latter value matches well with the experimentally obtained Kd of AtKAI2 for KAR1, which ranges from 4.6 to $148 \mu \mathrm{M}$ (Sun et al. 2020).

KAI2 has the catalytic triad residues, Ser95-Asp217His246, capable of hydrolyzing butanolide substrates (Nakamura et al. 2013; De Saint Germain et al. 2016; Yao et al. 2016). However, KAI2 hydrolysis activity for KARs has not been reported (Xu et al. 2016). Indeed, it is under discussion whether hydrolysis is required for signal transduction (Zhao 
Fig. 1 Homology models of HaKAI2s and their interaction with STLs. a and b Homology model of HaKAI2a (in red) and HaKAI2b (in blue). The overall $Z$ score (comprising dihedral, and $1 \mathrm{D}$ packing and $3 \mathrm{D}$ packing $Z$ scores) for the HAKAI2a and HaKAI $2 b$ models is -0.07 and -0.09 , respectively. c Chemical structures of karrikin1, 8-epixanthatin, and tomentosin. $\mathbf{d}$ and $\mathbf{e}$ The hydrophobic-binding pocket of HaKAI2a (d) and HaKAI2b (e) accommodates 8-epixanthatin (8-epi, red) and tomentosin (tom, green). The amino acid residues involved in the interaction with 8-epixanthatin and tomentosin are shown in yellow. $\mathbf{f}$ and $\mathbf{g}$ 8-Epixanthatin (f) and tomentosin (g) fit into the hydrophobic pockets of HaKAI2a and HaKAI2b. The hydrophobic amino acid residues in the pockets are marked in yellow

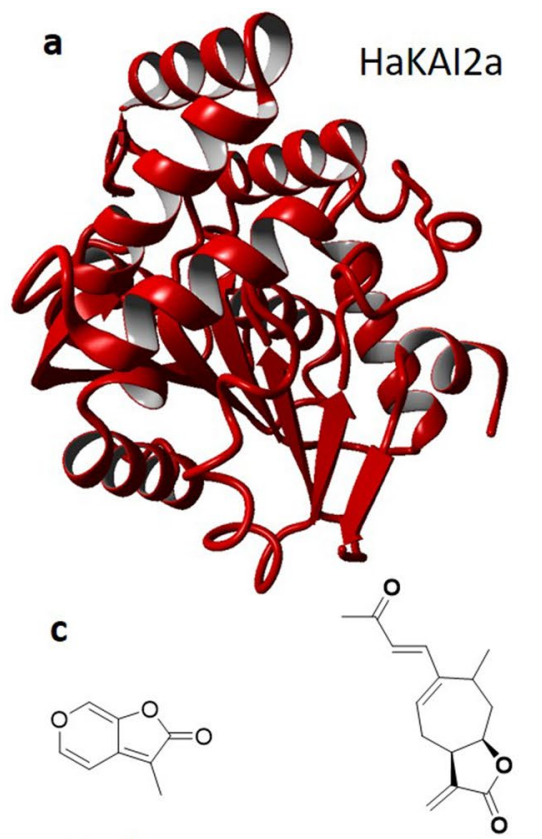

Karrikin 1

8-epixanthatin
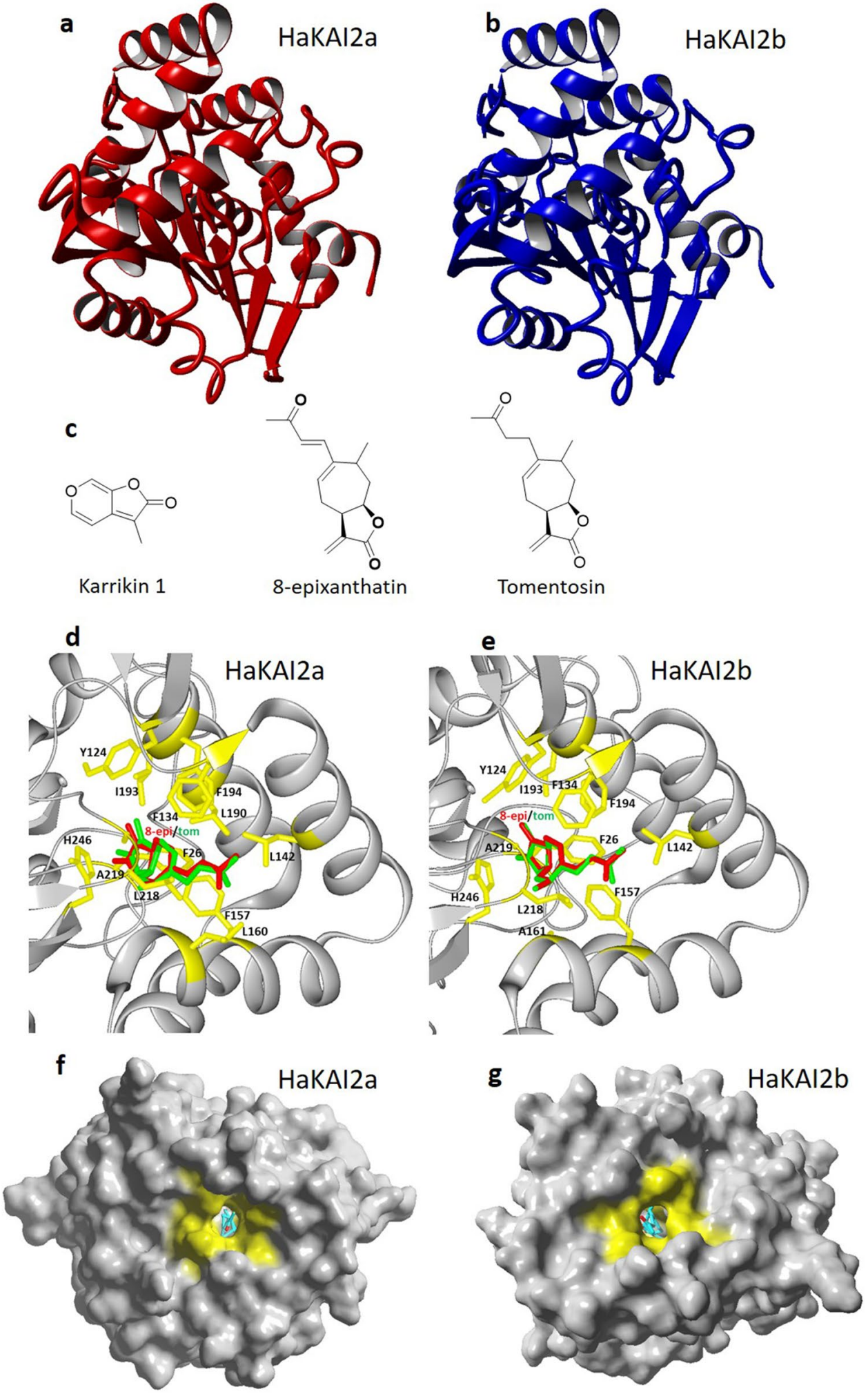

et al. 2013; Shabek et al. 2018; Seto et al. 2019; Yao and Waters 2020). Thus, KL may be a compound or a group of compounds that cannot undergo hydrolysis and share structural similarity with KARs. Our results suggest that STLs may be a member of this group. Sesquiterpenes have been detected in all vascular plants, and in many other organisms such as insects and fungi. Also Arabidopsis produces a large variety of sesquiterpenes (Tholl et al. 2005; Ro et al. 
2006). Oxidized derivatives of these sesquiterpenes could be potential candidates for KL, although, to our knowledge, STLs have not yet been reported in Arabidopsis.

With this study, we provide candidate ligands, consisting of known endogenous plant metabolites, that can bind to KAI2 with an affinity that is much higher than for the only known exogenous ligand KAR. This finding opens up a new path to elucidate the mysterious ligand of KAI2, an important open question in plant biology with large potential applications in agriculture. Our work supports the hypothesis of others that the STLs are a plant growth regulator involved in growth and development of sunflower. We show that this effect is likely due to the interaction with KAI2. Future experimental data should validate our computational work. Studies into metabolites, possibly sesquiterpenoid, with similar lactone ring structure in Arabidopsis and other flowering plants should further solve the long-standing enigma of the elusive $\mathrm{KL}$ in the rest of the plant kingdom.

Author contribution statement H.B. and M.R. designed the project, M.R. did the modelling, and M.R. and H.B. wrote the article.

Acknowledgements This work was supported by ERC Advanced grant CHEMCOMRHIZO (670211) (to H.B., and M.R.).

Open Access This article is licensed under a Creative Commons Attribution 4.0 International License, which permits use, sharing, adaptation, distribution and reproduction in any medium or format, as long as you give appropriate credit to the original author(s) and the source, provide a link to the Creative Commons licence, and indicate if changes were made. The images or other third party material in this article are included in the article's Creative Commons licence, unless indicated otherwise in a credit line to the material. If material is not included in the article's Creative Commons licence and your intended use is not permitted by statutory regulation or exceeds the permitted use, you will need to obtain permission directly from the copyright holder. To view a copy of this licence, visit http://creativecommons.org/licenses/by/4.0/.

\section{References}

Arai T, Toda Y, Kato K et al (2013) Artabolide, a novel polar auxin transport inhibitor isolated from Artemisia absinthium. Tetrahedron 69:7001-7005. https://doi.org/10.1016/j.tet.2013.06.052

Bouwmeester H, Li C, Thiombiano B, et al (2020) Adaptation of the lifecycle of root parasitic plants to their host. Germination is controlled by essential host signaling molecules. Plant Physiol (in press)

Chadwick M, Trewin H, Gawthrop F, Wagstaff C (2013) Sesquiterpenoids lactones: benefits to plants and people. Int J Mol Sci 14:12780-12805. https://doi.org/10.3390/ijms140612780

Conn CE, Nelson DC (2016) Evidence that KARRIKIN-INSENSITIVE2 (KAI2) receptors may perceive an unknown signal that is not karrikin or strigolactone. Front Plant Sci 6:1219. https:// doi.org/10.3389/fpls.2015.01219
Conn CE, Bythell-Douglas R, Neumann D et al (2015) Convergent evolution of strigolactone perception enabled host detection in parasitic plants. Science 349:540-543. https://doi.org/10.1126/ science.aab1140

De Saint GA, Clavé G, Badet-Denisot MA et al (2016) An histidine covalent receptor and butenolide complex mediates strigolactone perception. Nat Chem Biol 12:787-794. https://doi. org/10.1038/nchembio. 2147

Joel DM, Chaudhuri SK, Plakhine D et al (2011) Dehydrocostus lactone is exuded from sunflower roots and stimulates germination of the root parasite Orobanche cumana. Phytochemistry 72:624-634. https://doi.org/10.1016/j.phytochem.2011.01.037

Liu Q, Beyraghdar Kashkooli A, Manzano D et al (2018) Kauniolide synthase is a P450 with unusual hydroxylation and cyclization-elimination activity. Nat Commun 9:4657. https://doi. org/10.1038/s41467-018-06565-8

Nakamura H, Xue Y-L, Miyakawa T et al (2013) Molecular mechanism of strigolactone perception by DWARF14. Nat Commun 4:2613. https://doi.org/10.1038/ncomms3613

Nelson DC, Flematti GR, Riseborough J-A et al (2010) Karrikins enhance light responses during germination and seedling development in Arabidopsis thaliana. Proc Natl Acad Sci U S A 107:7095-7100. https://doi.org/10.1073/pnas.0911635107

Raupp FM, Spring O (2013) New sesquiterpene lactones from sunflower root exudate as germination stimulants for Orobanche cumana. J Agric Food Chem 61:10481-10487. https://doi. org/10.1021/jf402392e

Ro D-K, Ehlting J, Keeling CI et al (2006) Microarray expression profiling and functional characterization of AtTPS genes: duplicated Arabidopsis thaliana sesquiterpene synthase genes At4g13280 and At4g13300 encode root-specific and woundinducible (Z)- $\gamma$-bisabolene synthases. Arch Biochem Biophys 448:104-116. https://doi.org/10.1016/j.abb.2005.09.019

Seto Y, Yasui R, Kameoka H et al (2019) Strigolactone perception and deactivation by a hydrolase receptor DWARF14. Nat Commun 10:191. https://doi.org/10.1038/s41467-018-08124-7

Shabek N, Ticchiarelli F, Mao H et al (2018) Structural plasticity of D3-D14 ubiquitin ligase in strigolactone signalling. Nature 563:652-656. https://doi.org/10.1038/s41586-018-0743-5

Spring O, Hager A (1982) Inhibition of elongation growth by two sesquiterpene lactones isolated from Helianthus annuus $\mathrm{L}$. Planta 156:433-440. https://doi.org/10.1007/BF00393314

Spring O, Schmauder K, Lackus ND et al (2020) Spatial and developmental synthesis of endogenous sesquiterpene lactones supports function in growth regulation of sunflower. Planta 252:2. https://doi.org/10.1007/s00425-020-03409-y

Sun X-D, Ni M (2011) HYPOSENSITIVE TO LIGHT, an alpha/beta fold protein, acts downstream of ELONGATED HYPOCOTYL 5 to regulate seedling de-etiolation. Mol Plant 4:116-126. https ://doi.org/10.1093/mp/ssq055

Sun YK, Yao J, Scaffidi A et al (2020) Divergent receptor proteins confer responses to different karrikins in two ephemeral weeds. Nat Commun 11:1264. https://doi.org/10.1038/s41467-02014991-w

Tholl D, Chen F, Petri J et al (2005) Two sesquiterpene synthases are responsible for the complex mixture of sesquiterpenes emitted from Arabidopsis flowers. Plant J 42:757-771. https://doi. org/10.1111/j.1365-313X.2005.02417.x

Toh S, Holbrook-Smith D, Stogios PJ et al (2015) Structure-function analysis identifies highly sensitive strigolactone receptors in Striga. Science 350:203-207. https://doi.org/10.1126/scien ce. aac 9476

Ueda J, Toda Y, Kato K et al (2013) Identification of dehydrocostus lactone and 4-hydroxy- $\beta$-thujone as auxin polar transport inhibitors. Acta Physiol Plant 35:2251-2258 
Waters MT, Smith SM (2013) KAI2- and MAX2-mediated responses to karrikins and strigolactones are largely independent of HY5 in Arabidopsis seedlings. Mol Plant 6:63-75. https://doi. org/10.1093/mp/sss 127

Xu Y, Miyakawa T, Nakamura H et al (2016) Structural basis of unique ligand specificity of KAI2-like protein from parasitic weed Striga hermonthica. Sci Rep 6:31386. https://doi. org/10.1038/srep31386

Yao J, Waters MT (2020) Perception of karrikins by plants: a continuing enigma. J Exp Bot 71:1774-1781. https://doi.org/10.1093/ jxb/erz548

Yao R, Ming Z, Yan L et al (2016) DWARF14 is a non-canonical hormone receptor for strigolactone. Nature 536:469-473. https ://doi.org/10.1038/nature19073
Yokotani-Tomita K, Kato J, Yamada K et al (1999) 8-Epixanthatin, a light-induced growth inhibitor, mediates the phototropic curvature in sunflower (Helianthus annuиs) hypocotyls. Physiol Plant 106:326-330. https://doi.org/10.1034/j.1399-3054.1999.10631 $0 . \mathrm{x}$

Zhao L-H, Zhou XE, Wu Z-S et al (2013) Crystal structures of two phytohormone signal-transducing $\alpha / \beta$ hydrolases: karrikinsignaling KAI2 and strigolactone-signaling DWARF14. Cell Res 23:436-439. https://doi.org/10.1038/cr.2013.19

Publisher's Note Springer Nature remains neutral with regard to jurisdictional claims in published maps and institutional affiliations. 